\title{
EFEITO DO DESMAME PRECOCE NA CARCAÇA DE NOVILHOS TERMINADOS EM PASTAGEM E ABATIDOS AOS 24 MESES ${ }^{1}$
}

\author{
JOÃO RESTLE ${ }^{2}$, FABIANO NUNES VAZ ${ }^{3}$, LEONIR LUIZ PASCOAL ${ }^{4}$, DILCEU BORGES DE SENNA ${ }^{5}$, \\ RICARDO ZAMBARDA VAZ ${ }^{5}$ e GELSON LUIZ DIAS FEIJÓ 6
}

\begin{abstract}
RESUMO - Foram comparadas as características de carcaça de novilhos cruza Charolês x Nelore, desmamados aos 90 (T90) ou 210 (T210) dias. Utilizaram-se 63 terneiros, castrados, os quais foram terminados em pastagem cultivada de Avena strigosa + Lolium multiflorum + Trifolium vesiculosum e abatidos aos 24 meses de idade. Não houve diferença significativa em relação às características peso de fazenda ( $\mathrm{T} 90=437 \mathrm{~kg}$ e $\mathrm{T} 210=467 \mathrm{~kg}$ ), rendimento de carcaça quente (T90 $=53,33 \%$ e $\mathrm{T} 210=52,21 \%)$, peso de carcaça quente $(\mathrm{T} 90=233 \mathrm{~kg}$ e T210 $=244 \mathrm{~kg}$ ) e peso de carcaça fria $(\mathrm{T} 90=226 \mathrm{~kg}$ e T210 $=238 \mathrm{~kg}$ ). As carcaças dos dois tratamentos apresentaram conformação semelhante (T90 $=10,8$ pontos e $\mathrm{T} 210=11,0$ pontos). Também as porcentagens de dianteiro, costilhar e traseiro não diferiram significativamente entre os dois tratamentos, assim como as variáveis comprimento de carcaça, comprimento de perna, comprimento de braço, perímetro de braço e espessura de coxão. A espessura de gordura de cobertura não diferiu significativamente entre os dois grupos de carcaças (T90=2,56 mm e T210=2,27 mm). Os resultados mostraram que o desmame precoce aos 90 dias não afeta as características de carcaça de novilhos abatidos aos 24 meses de idade, desde que as condições de alimentação durante o período de recria e terminação tenham sido adequadas.
\end{abstract}

Termos para indexação: Charolês, conformação, desmame precoce, espessura de gordura, Nelore.

\section{EARLY WEANING EFFECT ON CARCASS OF STEERS FINISHED ON PASTURE AND SLAUGHTERED AT TWENTY-FOUR MONTHS}

\begin{abstract}
Carcass characteristics of Charolais x Nellore steers, weaned at two ages: 90 (T90) or 210 days (T210) were evaluated. Sixty-three castrated calves, finished on Avena strigosa + Lolium multiflorum + Trifolium vesiculosum pasture and slaughtered at twenty-four months of age, were used. No significant differences were observed between treatments for final weight (T90 $=437 \mathrm{~kg}$ and $\mathrm{T} 210=467 \mathrm{~kg}$ ), dressing percentage $(\mathrm{T} 90=53.33 \%$ and $\mathrm{T} 210=52.21 \%)$, hot carcass weight ( T90 $=233 \mathrm{~kg}$ and $\mathrm{T} 210=244 \mathrm{~kg}$ ) and cold carcass weight $(\mathrm{T} 90=226 \mathrm{~kg}$ and $\mathrm{T} 210=238 \mathrm{~kg}$ ). Carcasses of the two treatments showed similar conformation (T90=10.8 points and $\mathrm{T} 210=11.0$ points). Also the forequarter, side and hindquarter percentages were similar between treatments, likewise no significant differences were observed for carcass, leg and arm length, arm perimeter and cushion thickness. Subcutaneous fat thickness did not differ between the two carcass groups (T90 $=2.56 \mathrm{~mm}$ and $\mathrm{T} 210=2.27 \mathrm{~mm}$ ). Results demonstrate that early weaning at ninety days of age does not affect carcass traits of steers slaughtered at twenty-four months of age, if adequate nutrition is provided during growing and finishing periods.
\end{abstract}

Index terms: Charolais, conformation, early weaning, subcutaneous fat thickness, Nellore.

\footnotetext{
${ }^{1}$ Aceito para publicação em 8 de dezembro de 1998.

2 Eng. Agr., Ph.D., Prof. Titular, Dep. de Zootecnia da Universidade Federal de Santa Maria (UFSM), Campus Camobi, CEP 97119900 Santa Maria, RS. Bolsista do CNPq. E-mail: restle@infoway.com.br

${ }^{3}$ Zoot., Aluno do Curso de Mestrado, UFSM.

${ }^{4}$ Zoot., M.Sc., Prof. Assistente, Dep. de Zootecnia, UFSM.

${ }^{5}$ Zoot., M.Sc., Dep. de Zootecnia, UFSM.

${ }^{6}$ Méd. Vet., M.Sc., Embrapa-Centro Nacional de Pesquisa de Gado de Corte (CNPGC), Rodovia BR-262, km 4, CEP 79002-970 Campo Grande, MS.
}

\section{INTRODUÇÃo}

Uma vez que o Brasil tem o maior rebanho comercial de bovinos do mundo, é inadmissível que a produtividade da pecuária brasileira esteja em níveis tão inferiores à de países (Anuário..., 1996) que apresentam maiores restrições de recursos alimentares para os rebanhos. Segundo Pascoal \& Vaz (1997), em se tratando de melhoria dos índices de desfrute da pecuária brasileira, nenhum índice acarretaria melhor 
resultado que o aumento da taxa de repetição de cria dos ventres que, segundo os mesmos autores, pode ser alcançada com a adoção do desmame precoce dos terneiros, nas propriedades que apresentam índices de repetição de cria dos ventres inferior a $65 \%$.

Além de melhorar os índices de repetição de cria, o desmame precoce é importante para os rebanhos porque também melhora o estado corporal da vaca, conforme demonstrado no trabalho de Makarechian et al. (1988), em que a antecipação da idade ao desmame em um mês diminuiu a perda de peso das vacas durante o inverno de $0,480 \mathrm{~kg} /$ dia para $0,003 \mathrm{~kg} / \mathrm{dia}$.

Outros trabalhos também demonstraram a importância do desmame precoce. Desmamando terneiros aos 101 dias de idade, Moojen et al. (1994) verificaram aumento na taxa de aparecimento de cio pós-parto e melhora no estado corporal da vaca no outono. Barcellos et al. (1995), desterneirando vacas aos 70 ou 120 dias pós-parto, concluíram que a retirada do terneiro precocemente possibilitou um maior ganho de peso das mães no pós-desmame. Para outros autores, além de melhorar os índices de repetição de cria e estado corporal da vaca, o desmame precoce não prejudicou o desenvolvimento futuro do novilho ou novilha (Grimes \& Turner, 1991; Albospino \& Lobato, 1994; Kozloski et al., 1995).

Utilizando animais inteiros confinados, os trabalhos de Vaz et al. (1997) e Restle et al. (1999), demonstraram, em condições brasileiras, que é possível terminar animais desmamados precocemente para que sejam abatidos aos 14 meses de idade, com peso de carcaça e acabamento dentro dos limites exigidos pelos frigoríficos e legislações de incentivo ao abate de animais jovens (Rio Grande do Sul, 1995). No entanto, nesses trabalhos os animais desmamados precocemente foram confinados logo após o desmame e mantidos sob rações altamente energéticas até o abate aos 14 meses.

O objetivo deste trabalho foi estudar as características de carcaça de novilhos, ao se reduzir de 210 dias, idade em que normalmente se realizam os desmames no Brasil, para 90 dias, programando-se o abate dos animais aos 24 meses, após terminação em pastagem cultivada de estação fria.

\section{MATERIAL E MÉTODOS}

Este experimento foi conduzido no Departamento de Zootecnia da Universidade Federal de Santa Maria, de janeiro de 1990 - quando distribuiu-se aleatoriamente 63 terneiros Charolês x Nelore em um dos dois tratamentos: $\mathrm{T} 90=$ terneiros desmamados aos 90 dias, e $\mathrm{T} 210=$ terneiros desmamados aos 210 dias de idade - até o mês de outubro de 1991, quando foram abatidos os novilhos dos dois tratamentos.

Após o desmame, os terneiros do T90 foram alimentados por um período de 30 dias com capim-elefante (Pennisetum purpureum) picado mais $1 \mathrm{~kg}$ de farelo de soja/animal/dia, sendo em seguida mantidos em pastagem cultivada de papuã (Brachiaria plantaginea), até os sete meses de idade, sendo posteriormente levados para pastagem cultivada de aveia-preta (Avena strigosa) + azevém (Lolium multiflorum) + trevo vesiculoso (Trifolium vesiculosum). Após o desmame, os terneiros do T210 foram alimentados no cocho por um período de 30 dias, recebendo cana-de-açúcar (Saccharum officinarum) mais $1 \mathrm{~kg}$ de farelo de soja/animal/dia, e após levados para a mesma área de pastagem cultivada de inverno onde estavam os terneiros do T90. Durante o verão, os terneiros foram mantidos em campo nativo, e no segundo inverno os novilhos permaneceram novamente sobre pastagem cultivada de aveia + azevém + trevo vesiculoso, onde realizou-se a terminação.

Ao longo do período experimental, os animais receberam as mesmas condições de manejo e sanidade, sendo a castração realizada aos oito meses de idade. Quando os animais atingiram peso vivo médio de $450 \mathrm{~kg}$, foram levados para serem abatidos no Frigorífico Silva, localizado no mesmo município. Após um jejum de sólidos de 14 horas, e antes do embarque para o frigorífico, os animais foram pesados, obtendo-se o peso de fazenda.

Após o abate, as carcaças foram identificadas, pesadas e levadas para o resfriamento por aproximadamente 24 horas, a uma temperatura de $-2^{\circ} \mathrm{C}$. Decorrido esse tempo, as carcaças foram novamente pesadas, coletados os dados de comprimento de carcaça, de perna, de braço, espessura de coxão e perímetro de braço, conforme técnica descrita no trabalho de Muller (1987).

As carcaças também foram avaliadas quanto à conformação seguindo, uma escala de 1 a 18 pontos, e maturidade fisiológica (Muller, 1987), e medida a espessura de gordura subcutânea sobre a $12^{\mathrm{a}}$ costela, com ajuste do valor quando não era representativa da real cobertura de gordura da carcaça. Também foi medida a percentagem de corte dianteiro, traseiro (ou corte serrote) e costilhar. 
O delineamento experimental foi o inteiramente casualizado, com número desigual de repetições por tratamento. Os resultados foram submetidos à análise de variância, através do método dos quadrados mínimos e teste $\mathrm{F}$, utilizando o seguinte modelo matemático:

$Y_{i j}=\mu+T_{i}+e_{i j}$, em que:

$Y_{i j}=$ observação $\mathrm{j}$ do animal que recebeu tratamento $\mathrm{i}$;

$\mu=$ média geral;

$T_{i}=$ efeito do tratamento $\mathrm{i}$;

$e_{i j}=$ erro aleatório associado a cada observação.

Também foram calculados os coeficientes de correlação entre as variáveis dependentes dentro dos tratamentos, e verificada a probabilidade da correlação ser significativa a $5 \%$ pelo teste $t$.

\section{RESULTADOS E DISCUSSÃO}

Na Tabela 1 encontram-se os resultados da análise de variância e teste $\mathrm{F}$ das características estudadas. Na Tabela 2 são apresentados os resultados referentes a peso de fazenda, de carcaça, rendimento e quebra ao resfriamento. Observa-se que não houve diferença $(\mathrm{P}>0,05)$ entre os tratamentos quanto a essas características. A adequada nutrição dos animais dos dois tratamentos durante a recria e terminação foi o fator responsável por não verificar-se diferença no peso de fazenda ( $\mathrm{P}>0,0893)$ e pesos de carcaça quente $(\mathrm{P}>0,2831)$ e fria $(\mathrm{P}>0,2027)$.

O trabalho de Kubisch \& Makarechian (1987) utilizou 367 terneiros inteiros, desmamados aos $157 \mathrm{ou}$ 189 dias de idade. Embora pequena a diferença na idade ao desmame, os autores verificaram que os desmamados mais precocemente foram $14,3 \mathrm{~kg}$ mais leves ao abate que os desmamados com mais idade. Entretanto, Grimes \& Turner (1991) verificaram, em cinco anos de estudo, que o desmame aos 110 dias resultou em animais $14 \mathrm{~kg}$ mais pesados que aos 220 dias de idade.

Em condições brasileiras, Albospino \& Lobato (1994) não verificaram diferença significativa no peso de fazenda e carcaça quente de novilhos abatidos aos 26 meses, registrando peso vivo de $416,4 \mathrm{~kg}$ e $414,0 \mathrm{~kg}$, e peso de carcaça quente de $224,5 \mathrm{~kg}$ e $223,1 \mathrm{~kg}$, respectivamente, em novilhos desmamados aos 100 ou 150 dias. Cachapuz (1997) cita peso de carcaça quente de $233 \mathrm{~kg}$ e $226 \mathrm{~kg}$, respectivamente, em terneiros desmamados aos 90 e 205 dias. Restle et al. (1999) trabalhando com animais terminados em confinamento e abatidos aos 14 meses de idade também atribuem à adequada nutrição dos animais a semelhança no peso de fazenda e peso de

TABELA 1. Quadrados médios (QM) das análises de variância e teste $F$, nas características de carcaça de animais desmamados aos 90 ou 210 dias de idade.

\begin{tabular}{|c|c|c|c|c|c|c|}
\hline \multirow[t]{2}{*}{ Característica } & \multicolumn{2}{|c|}{ Graus de liberdade } & \multicolumn{2}{|c|}{ QM } & \multirow[t]{2}{*}{$\mathrm{F}$} & \multirow[t]{2}{*}{$\mathrm{R}^{2}$} \\
\hline & Modelo & Erro & Modelo & Erro & & \\
\hline Peso de fazenda & 1 & 61 & 14269 & 4786 & 2,98 & 0,0466 \\
\hline Peso de carcaça quente & 1 & 61 & 1660 & 1415 & 1,17 & 0,0189 \\
\hline Peso de carcaça fria & 1 & 61 & 2227 & 1343 & 1,66 & 0,0265 \\
\hline Rendimento de carcaça & 1 & 61 & 19,625 & 5,610 & 3,50 & 0,0542 \\
\hline Quebra ao resfriamento & 1 & 61 & 9,047 & 2,949 & 3,07 & 0,0479 \\
\hline Comprimento de carcaça & 1 & 61 & 213,47 & 57,42 & 3,72 & 0,0574 \\
\hline Comprimento de perna & 1 & 61 & 29,03 & 16,56 & 1,75 & 0,0279 \\
\hline Espessura de coxão & 1 & 61 & 3,580 & 2,531 & 1,41 & 0,0227 \\
\hline Comprimento de braço & 1 & 61 & 5,808 & 3,716 & 1,56 & 0,0250 \\
\hline Perímetro de braço & 1 & 61 & 7,097 & 6,108 & 1,16 & 0,0187 \\
\hline Conformação & 1 & 61 & 0,746 & 2,639 & 0,28 & 0,0046 \\
\hline Maturidade fisiológica & 1 & 61 & 0,001 & 0,605 & 0,00 & 0,0001 \\
\hline Espessura de gordura & 1 & 61 & 1,309 & 1,792 & 0,73 & 0,0118 \\
\hline Porcentagem de dianteiro & 1 & 61 & 0,834 & 1,290 & 0,65 & 0,0105 \\
\hline Porcentagem de costilhar & 1 & 61 & 0,017 & 0,792 & 0,02 & 0,0004 \\
\hline Porcentagem de traseiro & 1 & 61 & 0,613 & 1,426 & 0,43 & 0,0070 \\
\hline
\end{tabular}


carcaça. Nesse trabalho, os autores verificaram peso de carcaça quente de $232 \mathrm{~kg}$ e $221 \mathrm{~kg}$, respectivamente, em animais desmamados aos 72 dias ou 210 dias, e peso de carcaça fria de $224 \mathrm{~kg}$ e $214 \mathrm{~kg}$, na mesma ordem.

Ainda na Tabela 2, verifica-se que o rendimento de carcaça quente dos novilhos em função do peso de fazenda foi de $53,33 \%$ e $52,21 \%$, respectivamente, em T90 e T210 (P>0,0662). Albospino \& Lobato (1994) registraram o mesmo rendimento de carcaça quente $(56,1 \%)$ entre novilhos desmamados aos 100 ou 150 dias. Restle et al. (1999) verificaram que o rendimento de carcaça quente foi de $54,7 \%$ e $54,4 \%$, e o rendimento de carcaça fria foi de $52,8 \%$ e $52,7 \%$, respectivamente, em machos inteiros desmamados aos 72 ou 210 dias. Os mesmos autores concluíram que a teoria empírica de que animais desmamados precocemente teriam pior rendimento de carcaça que aqueles desmamados com mais idade, pelo maior volume do trato digestivo, não se mostrou verdadeira. No presente estudo, a quebra ocorrida durante as 24 horas de resfriamento foi de $3,25 \%$ e $2,50 \%$ ( $\mathrm{P}>0,0849$ ), respectivamente, em T90 e T210.

$\mathrm{Na}$ Tabela 3, são apresentados os resultados referentes a comprimento de carcaça, de perna, de braço, espessura de coxão, perímetro de braço, conformação e espessura de gordura subcutânea. Observa-se que essas características, que refletem o de-

TABELA 2. Média e desvio-padrão (DP) de peso de fazenda e peso de carcaça quente e de carcaça fria, rendimento e quebra no resfriamento da carcaça de animais desmamados aos 90 ou 210 dias de idade.

\begin{tabular}{|c|c|c|c|c|c|c|}
\hline \multirow[t]{2}{*}{ Parâmetro } & \multicolumn{2}{|c|}{$\mathrm{T} 90$} & \multicolumn{2}{|c|}{$\mathrm{T} 210$} & \multirow[t]{2}{*}{$\mathrm{CV}^{1}(\%)$} & \multirow{2}{*}{$\begin{array}{c}\text { Probabi- } \\
\text { lidade }\end{array}$} \\
\hline & Média & DP & Média & DP & & \\
\hline Peso de fazenda $(\mathrm{kg})$ & 437 & 12,2 & 467 & 12,4 & 15,31 & 0,0893 \\
\hline Peso de carcaça quente $(\mathrm{kg})$ & 233 & 6,7 & 244 & 6,8 & 15,78 & 0,2831 \\
\hline Peso de carcaça fria $(\mathrm{kg})$ & 226 & 6,5 & 238 & 6,6 & 15,83 & 0,2027 \\
\hline Rendimento carcaça quente (\%) & 53,33 & 0,42 & 52,21 & 0,43 & 4,49 & 0,0662 \\
\hline Quebra ao resfriamento (\%) & 3,25 & 0,30 & 2,50 & 0,31 & 59,58 & 0,0849 \\
\hline
\end{tabular}

${ }^{1}$ Coeficiente de variação.

TABELA 3. Média e desvio-padrão (DP) de medidas de carcaça, conformação, maturidade fisiológica e espessura de gordura de cobertura na carcaça de animais desmamados aos 90 ou 210 dias de idade.

\begin{tabular}{|c|c|c|c|c|c|c|}
\hline \multirow[t]{2}{*}{ Parâmetro } & \multicolumn{2}{|c|}{ T90 } & \multicolumn{2}{|c|}{$\mathrm{T} 210$} & \multirow[t]{2}{*}{$\mathrm{CV}^{1}(\%)$} & \multirow{2}{*}{$\begin{array}{c}\text { Probabi- } \\
\text { lidade }\end{array}$} \\
\hline & Média & DP & Média & DP & & \\
\hline Comprim. carcaça $(\mathrm{cm})$ & 118,1 & 1,34 & 121,8 & 1,36 & 6,32 & 0,0585 \\
\hline Comprim. perna (cm) & 71,1 & 0,72 & 72,5 & 0,73 & 5,67 & 0,1904 \\
\hline Espessura coxão (cm) & 25,3 & 0,28 & 25,8 & 0,29 & 6,24 & 0,2390 \\
\hline Comprim. braço $(\mathrm{cm})$ & 40,3 & 0,34 & 40,9 & 0,35 & 4,74 & 0,2160 \\
\hline Perímetro braço $(\mathrm{cm})$ & 34,3 & 0,44 & 35,0 & 0,44 & 7,13 & 0,2853 \\
\hline Conformação ${ }^{2}$ & 10,8 & 0,29 & 11,0 & 0,29 & 14,96 & 0,5967 \\
\hline Maturidade fisiológica & 13,2 & 0,14 & 13,2 & 0,14 & 5,88 & 0,9714 \\
\hline Esp. gordura subcut. (mm) & 2,56 & 0,24 & 2,27 & 0,24 & 55,30 & 0,3961 \\
\hline
\end{tabular}

${ }^{1}$ Coeficiente de variação.

${ }^{2}$ Valor $10=$ boa menos; 11 = boa típica; $12=$ boa mais. 
senvolvimento muscular (espessura de coxão e perímetro de braço) e ósseo da carcaça (comprimento de carcaça, de perna e de braço), não apresentaram diferença significativa entre os tratamentos. Utilizando machos inteiros terminados em confinamento, Restle et al. (1999) não verificaram diferença significativa quanto a essas mesmas características. Cachapuz (1997) verificou comprimento de carcaça de $126,0 \mathrm{~cm}$ e $125,5 \mathrm{~cm}$ em terneiros desmamados aos 90 e 205 dias, respectivamente.

Também na Tabela 3 são apresentados os resultados referentes à avaliação da conformação das carcaças. Os animais dos dois tratamentos $(\mathrm{P}>0,5967)$ apresentaram carcaças com classificação "boa típica" (T90=10,8 pontos e T210=11,0 pontos). Similaridade na conformação de carcaça foi verificada por Restle et al. (1999), embora tenham verificado conformação de carcaça classificada como "boa mais" ( 11,8 pontos) nos animais desmamados aos 72 dias, e "boa típica" (11,3 pontos) aos 210 dias.

A espessura de gordura sobre a $12^{\mathrm{a}}$ costela foi de 2,56 mm e 2,27 mm ( $>>0,3961)$, respectivamente, em T90 e T210 (Tabela 3). Grimes \& Turner (1991), confinando animais até que atingissem $8,9 \mathrm{~mm}$ sobre a $12^{\mathrm{a}}$ costela, verificaram que os terneiros desmamados aos 110 dias foram 11 dias mais jovens ao abate do que os que permaneceram ao pé da vaca até os 220 dias. Restle et al. (1999) não verificaram diferença na espessura de gordura sobre a carcaça, cuja média entre animais desmamados precocemente ou não foi de 4,4 mm, valor superior ao do presente estudo, provavelmente pelo fato de naquele terem sido utilizados animais Hereford x Nelore e maior nível alimentar oferecido aos animais em confinamento para que possibilitasse o abate aos 14 meses de idade, embora os animais fossem inteiros.

A Tabela 4 apresenta os resultados referentes à porcentagem dos três cortes comerciais da carcaça. Pode-se verificar que os percentuais determinados para dianteiro, costilhar e traseiro especial foram de $36,70 \%, 12,50 \%$ e $50,80 \%$ em T90, e $36,93 \%, 12,47 \%$ e $50,60 \%$ em T210, e que nenhuma dessas características foi significativamente diferente. Com animais abatidos aos 26 meses e terminados em pastagem, Albospino \& Lobato (1994) não verificaram diferença significativa em porcentagem dos três cortes co- merciais da carcaça. No entanto, Restle et al. (1999) verificaram que machos inteiros abatidos aos 14 meses apresentaram maior porcentagem de costilhar quando o desmame ocorreu aos 72 dias $(12,8 \%)$ do que animais desmamados aos 210 dias de idade $(12,0 \%)$.

Na Tabela 5 é mostrado o comportamento das variáveis no T90, por meio do cálculo dos coeficientes de correlação de Pearson. Verifica-se que o peso de fazenda teve coeficiente de correlação alto e significativo com comprimento de carcaça, comprimento de perna, espessura de coxão, perímetro de braço e conformação. Essas correlações seguiram as tendências gerais encontradas em diferentes categorias de bovinos (Muller, 1987). Restle et al. (1999), trabalhando com animais desmamados aos 72 dias de idade verificaram coeficientes de correlação significativo entre peso de fazenda e as características de comprimento de carcaça, de perna e de braço, espessura de coxão e perímetro de braço, mas não em peso de fazenda e conformação.

A correlação entre peso de fazenda e espessura de gordura foi de $-0,07$ ( $\mathrm{P}>0,7316$ ), indicando que os animais mais pesados não foram necessariamente os de melhor acabamento. Essa característica deveu-se ao fato de as composições genéticas utilizadas não apresentarem precocidade em deposição de gordura (Restle et al., 1995a, 1995b, 1997). Utilizando-se de animais Braford, uma raça mais precoce em deposição de gordura, Restle et al. (1999) verificaram um coeficiente de correlação entre essas características um pouco maior $(\mathrm{r}=0,30)$, mas não-significativo.

$\mathrm{Na}$ Tabela 5 ainda pode-se verificar que o coeficiente de correlação não foi significativo entre peso de fazenda e rendimento de carcaça $(r=0,32$; $\mathrm{P}>0,0890$ ), no entanto foi alto e significativo entre peso de carcaça e rendimento $(\mathrm{r}=0,62 ; \mathrm{P}<0,0004)$. Tais resultados não foram verificados no trabalho de Restle et al. (1999), que encontraram um coeficiente de correlação entre essas características de 0,22. Assim como o peso de fazenda, o peso de carcaça foi significativamente correlacionado com a conformação de carcaça e teve baixa correlação com a espessura de gordura.

Os animais desmamados aos 90 dias de idade apresentaram correlação significativa entre rendimento de carcaça e espessura de gordura $(r=0,40$; 
$\mathrm{P}<0,0322)$. Os coeficientes de correlação também foram significativos entre rendimento de carcaça e todas as medidas de desenvolvimento muscular da carcaça (espessura de coxão, perímetro de braço e conformação). Trabalhando com animais desmamados aos 72 dias de idade, Restle et al. (1999) não verificaram correlação significativa entre rendimento e espessura de coxão e perímetro de braço, mas veri- ficaram valores de correlação significativos entre rendimento de carcaça quente e conformação $(r=0,63)$.

Na Tabela 6 são apresentados os coeficientes de correlação entre as variáveis estudadas no T210. Assim como no T90, o peso de fazenda foi significativamente correlacionado com as medidas métricas da carcaça. No entanto, a correlação não foi significativa entre peso de fazenda e conformação.

TABELA 4. Média e desvio-padrão (DP) de peso e porcentagem dos três cortes comerciais da carcaça de animais desmamados aos 72 ou 210 dias de idade.

\begin{tabular}{|c|c|c|c|c|c|c|}
\hline \multirow[t]{2}{*}{ Parâmetro } & \multicolumn{2}{|c|}{ T90 } & \multicolumn{2}{|c|}{$\mathrm{T} 210$} & \multirow[t]{2}{*}{$\mathrm{CV}^{1}(\%)$} & \multirow{2}{*}{$\begin{array}{c}\text { Probabi- } \\
\text { lidade }\end{array}$} \\
\hline & Média & DP & Média & DP & & \\
\hline Porcentagem de dianteiro & 36,70 & 0,20 & 36,93 & 0,20 & 3,06 & 0,4246 \\
\hline Porcentagem de costilhar & 12,50 & 0,16 & 12,47 & 0,16 & 6,88 & 0,8843 \\
\hline Porcentagem de traseiro & 50,80 & 0,21 & 50,60 & 0,21 & 2,37 & 0,5430 \\
\hline
\end{tabular}

${ }^{1}$ Coeficiente de variação.

TABELA 5. Coeficientes de correlação entre as variáveis estudadas da carcaça de animais desmamados aos 90 dias de idade.

\begin{tabular}{|c|c|c|c|c|c|c|c|c|c|c|c|c|}
\hline Variável & & PCQ & RCQ & QR & MF & $\mathrm{CC}$ & $\mathrm{CP}$ & $\mathrm{EC}$ & $\mathrm{CB}$ & PB & Co & $\mathrm{EG}^{1}$ \\
\hline Peso de fazenda & $\begin{array}{c}\mathrm{r} \\
\text { Prob. }\end{array}$ & $\begin{array}{c}0,94 \\
0,0001\end{array}$ & $\begin{array}{c}0,32 \\
0,0890\end{array}$ & $\begin{array}{c}-0,28 \\
0,1346\end{array}$ & $\begin{array}{c}-0,34 \\
0,0716\end{array}$ & $\begin{array}{c}0,89 \\
0,0001\end{array}$ & $\begin{array}{c}0,77 \\
0,0001\end{array}$ & $\begin{array}{c}0,61 \\
0,0005\end{array}$ & $\begin{array}{c}0,76 \\
0,0001\end{array}$ & $\begin{array}{c}0,74 \\
0,0001\end{array}$ & $\begin{array}{c}0,45 \\
0,0147\end{array}$ & $\begin{array}{c}-0,07 \\
0,7316\end{array}$ \\
\hline $\begin{array}{l}\text { Peso carc. quente } \\
\text { (PCQ) }\end{array}$ & $\begin{array}{c}\mathrm{r} \\
\text { Prob. }\end{array}$ & & $\begin{array}{c}0,62 \\
0,0004\end{array}$ & $\begin{array}{c}-0,07 \\
0,7331\end{array}$ & $\begin{array}{c}-0,30 \\
0,1178\end{array}$ & $\begin{array}{c}0,79 \\
0,0001\end{array}$ & $\begin{array}{c}0,76 \\
0,0001\end{array}$ & $\begin{array}{c}0,67 \\
0,0001\end{array}$ & $\begin{array}{c}0,71 \\
0,0001\end{array}$ & $\begin{array}{c}0,80 \\
0,0001\end{array}$ & $\begin{array}{c}0,52 \\
0,0039\end{array}$ & $\begin{array}{c}0,09 \\
0,6543\end{array}$ \\
\hline $\begin{array}{l}\text { Rend. carc. quente } \\
\text { (RCQ) }\end{array}$ & $\begin{array}{c}\mathrm{r} \\
\text { Prob. }\end{array}$ & & & $\begin{array}{c}0,46 \\
0,0130\end{array}$ & $\begin{array}{c}-0,07 \\
0,7146\end{array}$ & $\begin{array}{c}0,12 \\
0,5229\end{array}$ & $\begin{array}{c}0,34 \\
0,0756\end{array}$ & $\begin{array}{c}0,45 \\
0,0147\end{array}$ & $\begin{array}{c}0,21 \\
0,2784\end{array}$ & $\begin{array}{c}0,56 \\
0,0015\end{array}$ & $\begin{array}{c}0,45 \\
0,0137\end{array}$ & $\begin{array}{c}0,40 \\
0,0322\end{array}$ \\
\hline $\begin{array}{l}\text { Quebra resfriam. } \\
\text { (QR) }\end{array}$ & $\begin{array}{c}\mathrm{R} \\
\text { Prob. }\end{array}$ & & & & $\begin{array}{c}0,16 \\
0,3946\end{array}$ & $\begin{array}{c}-0,30 \\
0,1162\end{array}$ & $\begin{array}{c}-0,20 \\
0,3050\end{array}$ & $\begin{array}{c}-0,00 \\
0,9841\end{array}$ & $\begin{array}{l}-0,26 \\
0,1745\end{array}$ & $\begin{array}{c}-0,12 \\
0,5192\end{array}$ & $\begin{array}{c}-0,05 \\
0,8022\end{array}$ & $\begin{array}{c}0,20 \\
0,2933\end{array}$ \\
\hline $\begin{array}{l}\text { Matur. fisiológica } \\
(\mathrm{MF})\end{array}$ & $\begin{array}{c}\mathrm{r} \\
\text { Prob. }\end{array}$ & & & & & $\begin{array}{c}-0,34 \\
0,0734\end{array}$ & $\begin{array}{c}-0,39 \\
0,0390\end{array}$ & $\begin{array}{c}-0,11 \\
0,5686\end{array}$ & $\begin{array}{c}-0,36 \\
0,0521\end{array}$ & $\begin{array}{c}-0,37 \\
0,0488\end{array}$ & $\begin{array}{c}-0,18 \\
0,3597\end{array}$ & $\begin{array}{c}-0,04 \\
0,8420\end{array}$ \\
\hline $\begin{array}{l}\text { Compr. carcaça } \\
\text { (CC) }\end{array}$ & $\begin{array}{c}\mathrm{r} \\
\text { Prob. }\end{array}$ & & & & & & $\begin{array}{c}0,67 \\
0,0001\end{array}$ & $\begin{array}{c}0,44 \\
0,0159\end{array}$ & $\begin{array}{c}0,75 \\
0,0001\end{array}$ & $\begin{array}{c}0,60 \\
0,0006\end{array}$ & $\begin{array}{c}0,25 \\
0,1894\end{array}$ & $\begin{array}{c}-0,21 \\
0,2720\end{array}$ \\
\hline $\begin{array}{l}\text { Compr. perna } \\
\text { (CP) }\end{array}$ & $\begin{array}{c}\mathrm{r} \\
\text { Prob. }\end{array}$ & & & & & & & $\begin{array}{c}0,45 \\
0,0145\end{array}$ & $\begin{array}{c}0,76 \\
0,0001\end{array}$ & $\begin{array}{c}0,74 \\
0,0001\end{array}$ & $\begin{array}{c}0,59 \\
0,0007\end{array}$ & $\begin{array}{c}-0,13 \\
0,4964\end{array}$ \\
\hline $\begin{array}{l}\text { Espess. coxão } \\
\text { (EC) }\end{array}$ & $\begin{array}{c}\mathrm{r} \\
\text { Prob. }\end{array}$ & & & & & & & & $\begin{array}{c}0,39 \\
0,0362\end{array}$ & $\begin{array}{c}0,56 \\
0,0017\end{array}$ & $\begin{array}{c}0,14 \\
0,4581\end{array}$ & $\begin{array}{c}0,02 \\
0,9321\end{array}$ \\
\hline $\begin{array}{l}\text { Compr. braço } \\
\text { (CB) }\end{array}$ & $\begin{array}{c}\mathrm{r} \\
\text { Prob. }\end{array}$ & & & & & & & & & $\begin{array}{c}0,71 \\
0,0001\end{array}$ & $\begin{array}{c}0,39 \\
0,0340\end{array}$ & $\begin{array}{c}-0,13 \\
0,4887\end{array}$ \\
\hline $\begin{array}{l}\text { Perím. braço } \\
\text { (PB) }\end{array}$ & $\begin{array}{c}\mathrm{r} \\
\text { Prob. }\end{array}$ & & & & & & & & & & $\begin{array}{c}0,55 \\
0,0021\end{array}$ & $\begin{array}{c}-0,01 \\
0,9537\end{array}$ \\
\hline $\begin{array}{l}\text { Conformação } \\
\text { (Co) }\end{array}$ & $\begin{array}{c}\mathrm{r} \\
\text { Prob. }\end{array}$ & & & & & & & & & & & $\begin{array}{c}0,18 \\
0,3567\end{array}$ \\
\hline
\end{tabular}

\footnotetext{
${ }^{1}$ Espessura de gordura.
} 
TABELA 6. Coeficientes de correlação entre as variáveis estudadas da carcaça de animais desmamados aos 210 dias de idade.

\begin{tabular}{|c|c|c|c|c|c|c|c|c|c|c|c|c|}
\hline Variável & & PCQ & RCQ & QR & MF & $\mathrm{CC}$ & $\mathrm{CP}$ & $\mathrm{EC}$ & $\mathrm{CB}$ & $\mathrm{PB}$ & Co & $\mathrm{EG}^{1}$ \\
\hline Peso de fazenda & $\begin{array}{c}\mathrm{r} \\
\text { Prob. }\end{array}$ & $\begin{array}{c}0,94 \\
0,0001\end{array}$ & $\begin{array}{c}0,03 \\
0,8907\end{array}$ & $\begin{array}{c}-0,03 \\
0,7404\end{array}$ & $\begin{array}{c}0,16 \\
0,4306\end{array}$ & $\begin{array}{c}0,89 \\
0,0001\end{array}$ & $\begin{array}{c}0,37 \\
0,0510\end{array}$ & $\begin{array}{c}0,51 \\
0,0057\end{array}$ & $\begin{array}{c}0,58 \\
0,0012\end{array}$ & $\begin{array}{c}0,87 \\
0,0001\end{array}$ & $\begin{array}{c}0,33 \\
0,0847\end{array}$ & $\begin{array}{c}0,18 \\
0,3585\end{array}$ \\
\hline $\begin{array}{l}\text { Peso carc. quente } \\
\text { (PCQ) }\end{array}$ & $\begin{array}{c}\mathrm{r} \\
\text { Prob. }\end{array}$ & & $\begin{array}{c}0,36 \\
0,0563\end{array}$ & $\begin{array}{c}0,07 \\
0,7404\end{array}$ & $\begin{array}{c}0,07 \\
0,7177\end{array}$ & $\begin{array}{c}0,91 \\
0,0001\end{array}$ & $\begin{array}{c}0,44 \\
0,0190\end{array}$ & $\begin{array}{c}0,47 \\
0,0122\end{array}$ & $\begin{array}{c}0,63 \\
0,0004\end{array}$ & $\begin{array}{c}0,89 \\
0,0001\end{array}$ & $\begin{array}{c}0,32 \\
0,0971\end{array}$ & $\begin{array}{c}0,25 \\
0,1946\end{array}$ \\
\hline $\begin{array}{l}\text { Rend. carc. quente } \\
\text { (RCQ) }\end{array}$ & $\begin{array}{c}\mathrm{r} \\
\text { Prob. }\end{array}$ & & & $\begin{array}{c}0,26 \\
0,1876\end{array}$ & $\begin{array}{c}-0,22 \\
0,2508\end{array}$ & $\begin{array}{c}0,26 \\
0,1814\end{array}$ & $\begin{array}{c}0,30 \\
0,1150\end{array}$ & $\begin{array}{c}0,01 \\
0,9719\end{array}$ & $\begin{array}{c}0,30 \\
0,1209\end{array}$ & $\begin{array}{c}0,24 \\
0,2256\end{array}$ & $\begin{array}{c}-0,03 \\
0,8847\end{array}$ & $\begin{array}{c}0,21 \\
0,2901\end{array}$ \\
\hline $\begin{array}{l}\text { Quebra resfriam. } \\
\text { (QR) }\end{array}$ & $\begin{array}{c}\mathrm{R} \\
\text { Prob. }\end{array}$ & & & & $\begin{array}{c}-0,16 \\
0,4115\end{array}$ & $\begin{array}{c}0,10 \\
0,6226\end{array}$ & $\begin{array}{c}0,50 \\
0,0063\end{array}$ & $\begin{array}{c}-0,10 \\
0,6125\end{array}$ & $\begin{array}{c}0,09 \\
0,6648\end{array}$ & $\begin{array}{c}0,06 \\
0,7606\end{array}$ & $\begin{array}{c}0,05 \\
0,8127\end{array}$ & $\begin{array}{c}0,03 \\
0,8766\end{array}$ \\
\hline $\begin{array}{l}\text { Matur. fisiológica } \\
\text { (MF) }\end{array}$ & $\begin{array}{c}\mathrm{r} \\
\text { Prob. }\end{array}$ & & & & & $\begin{array}{c}0,10 \\
0,5958\end{array}$ & $\begin{array}{c}-0,04 \\
0,8295\end{array}$ & $\begin{array}{c}0,16 \\
0,4194\end{array}$ & $\begin{array}{c}-0,03 \\
0,8945\end{array}$ & $\begin{array}{c}0,09 \\
0,6603\end{array}$ & $\begin{array}{c}0,05 \\
0,7907\end{array}$ & $\begin{array}{l}-0,00 \\
0,9867\end{array}$ \\
\hline $\begin{array}{l}\text { Compr. carcaça } \\
\text { (CC) }\end{array}$ & $\begin{array}{c}\mathrm{r} \\
\text { Prob. }\end{array}$ & & & & & & $\begin{array}{c}0,47 \\
0,0111\end{array}$ & $\begin{array}{c}0,44 \\
0,0180\end{array}$ & $\begin{array}{c}0,65 \\
0,0002\end{array}$ & $\begin{array}{c}0,81 \\
0,0001\end{array}$ & $\begin{array}{c}0,07 \\
0,7093\end{array}$ & $\begin{array}{c}0,11 \\
0,5845\end{array}$ \\
\hline $\begin{array}{l}\text { Compr. perna } \\
\text { (CP) }\end{array}$ & $\begin{array}{c}\mathrm{r} \\
\text { Prob. }\end{array}$ & & & & & & & $\begin{array}{c}0,18 \\
0,3523\end{array}$ & $\begin{array}{c}0,36 \\
0,0563\end{array}$ & $\begin{array}{c}0,41 \\
0,0286\end{array}$ & $\begin{array}{c}-0,12 \\
0,5272\end{array}$ & $\begin{array}{c}0,34 \\
0,0814\end{array}$ \\
\hline $\begin{array}{l}\text { Espess. coxão } \\
\text { (EC) }\end{array}$ & $\begin{array}{c}\mathrm{r} \\
\text { Prob. }\end{array}$ & & & & & & & & $\begin{array}{c}0,16 \\
0,4068\end{array}$ & $\begin{array}{c}0,37 \\
0,0513\end{array}$ & $\begin{array}{c}0,01 \\
0,9746\end{array}$ & $\begin{array}{c}0,04 \\
0,8551\end{array}$ \\
\hline $\begin{array}{l}\text { Compr. braço } \\
\text { (CB) }\end{array}$ & $\begin{array}{c}r \\
\text { Prob. }\end{array}$ & & & & & & & & & $\begin{array}{c}0,66 \\
0,0001\end{array}$ & $\begin{array}{c}-0,01 \\
0,9489\end{array}$ & $\begin{array}{c}-0,05 \\
0,8030\end{array}$ \\
\hline $\begin{array}{l}\text { Perím. braço } \\
\text { (PB) }\end{array}$ & $\begin{array}{c}r \\
\text { Prob. }\end{array}$ & & & & & & & & & & $\begin{array}{c}0,38 \\
0,0482\end{array}$ & $\begin{array}{c}0,15 \\
0,4518\end{array}$ \\
\hline $\begin{array}{l}\text { Conformação } \\
\text { (Co) }\end{array}$ & $\begin{array}{c}\mathrm{r} \\
\text { Prob. }\end{array}$ & & & & & & & & & & & $\begin{array}{c}0,12 \\
0,5504\end{array}$ \\
\hline
\end{tabular}

${ }^{1}$ Espessura de gordura.

A mesma tendência do peso de fazenda foi verificada em peso de carcaça. Na Tabela 6 também verifica-se que, ao contrário do T90, o rendimento de carcaça não esteve correlacionado com as características de musculosidade da carcaça e espessura de gordura. Tais resultados estão de acordo com os de Restle et al. (1999), que verificaram coeficiente de correlação significativo entre rendimento de carcaça quente e conformação quando o desmame ocorreu aos 72 dias $(r=0,63)$, e decresceu aos 210 dias $(r=0,39)$.

\section{CONCLUSÕES}

1. A redução da idade ao desmame dos terneiros de 210 dias para 90 dias de idade não afeta as características de peso vivo, peso de carcaça e rendimento de carcaça de animais terminados em pastagem cultivada de inverno e abatidos aos 24 meses, desde que manejados de modo que a alimentação não seja restrita durante as fases de recria e terminação.

2. As medidas de desenvolvimento da carcaça e porcentagem dos cortes comerciais não apresentam diferença significativa entre novilhos desmamados precocemente ou não, quando o abate ocorre aos 24 meses de idade.

3. Existe correlação entre as características de desenvolvimento muscular da carcaça e rendimento de carcaça, quando a idade ao desmame ocorre aos 90 dias.

\section{AGRADECIMENTO}

Ao Prof. José Henrique Souza da Silva, pela valiosa orientação nas análises estatísticas. 


\section{REFERÊNCIAS}

ALBOSPINO, B.H.J.C.; LOBATO, J.F.P. Efeitos do desmame precoce de bezerros no desempenho até os 24-26 meses de idade. Revista da Sociedade Brasileira de Zootecnia, Viçosa, v.23, n.4, p.565-575, jul./ago. 1994.

ANUÁRIO ESTATÍSTICO DA PRODUÇÃO ANIMAL 96. São Paulo: Argos Comunicação, 1996. 312p.

BARCELLOS, J.O.J.; LOBATO, J.F.P.; SILVA, M.D. Desempenho ponderal de vacas e terneiros de corte submetidos ao desmame aos 70 ou 120 dias de idade. In: REUNIÃO ANUAL DA SOCIEDADE BRASILEIRA DE ZOOTECNIA, 32., 1995, Brasília. Anais. Brasília: SBZ, 1995. p.426-427.

CACHAPUZ, J.M.S. Experiências com desmame aos 90 e 60 dias. 2.ed. Porto Alegre: EMATER-RS, 1997. $52 \mathrm{p}$.

GRIMES, J.F.; TURNER, T.B. Early weaning of fall-born beef calves. 2. Postweaning performance of early and normal weaned calves. Journal of Production Agriculture, Columbus, v.4, n.4, p.468-471, Apr. 1991.

KOZLOSKI, G.V.; BONNECARRÈRE SANCHEZ, L.M.; BOETCHER, A. Alimentação de terneiros com dietas ricas em grãos e suplementados com uréia ou farinha de carne. I. Ganho de peso e consumo de alimento. In: REUNIÃO ANUAL DA SOCIEDADE BRASILEIRA DE ZOOTECNIA, 32., 1995, Brasília. Anais. Brasília: SBZ, 1995. p.201-202.

KUBISCH, H.M.; MAKARECHIAN, M. Effects of date of weaning on postweaning performance of bull calves of three breed groups. Canadian Journal of Animal Science, Lacombe, v.67, n.4, p.941-949, Apr. 1987.

MAKARECHIAN, M.; KUBISCH, H.M.; PRICE, M.A. Effects of date of weaning on subsequent performance of beef cows and their female calves. Canadian Journal of Animal Science, Lacombe, v.68, n.4, p.1035-1040, Apr. 1988.

MOOJEN, J.G.; RESTLE, J.; MOOJEN, E.L. Efeito da época da desmama e da pastagem no desempenho de vacas e terneiros de corte. 1. Desempenho das vacas. Ciência Rural, Santa Maria, v.24, n.2, p.393-397, abr./jun. 1994.
MULLER, L. Normas para avaliação de carcaças e concurso de carcaças de novilhos. 2.ed. Santa Maria: UFSM, Imprensa Universitária, 1987. 31p. (Publicação, n.1-DZ).

PASCOAL, L.L.; VAZ, F.N. Desmame precoce aos sessenta dias. In: RESTLE, J.; BRONDANI, I.L.; PASCOAL, L.L.; LUPATINI, G.C.; VAZ, F.N. Técnicas avançadas na recria e engorda de bovinos de corte. Santa Maria: UFSM, 1997. p.36-50.

RESTLE, J.; FELTEN, H.G.; VAZ, F.N. Efeito de raça e heterose para características quantitativas da carcaça de novilhos de 24 meses de idade terminados em confinamento. In: REUNION LATINOAMERICANA DE PRODUCCIÓN ANIMAL, 14., 1995, Mar del Plata. Anais. Balcarce: ALPA, 1995a. v.15, n.3/4, p.857-859.

RESTLE, J.; FELTEN, H.G.; VAZ, F.N.; MULLER, L. Efeito de raça e heterose para qualidade da carcaça e da carne de novilhos terminados em confinamento. In: REUNION LATINOAMERICANA DE PRODUCCIÓN ANIMAL, 14., 1995, Mar del Plata. Anais. Balcarce: ALPA, 1995b. v.15, n.3/4, p.854856.

RESTLE, J.; KEPLIN, L.A.S.; VAZ, F.N. Características quantitativas da carcaça de novilhos Charolês, abatidos com diferentes pesos. Pesquisa Agropecuária Brasileira, Brasília, v.32, n.8, p.851856. ago. 1997.

RESTLE, J.; VAZ, F.N.; BRONDANI, I.L.; GONÇALVES, J.M.; ANDREATTA, E. Estudo da carcaça de machos Braford desmamados aos 72 ou 210 dias, abatidos aos cartorze meses. Pesquisa Agropecuária Brasileira, Brasília, v. 34, n.11, p.21372144, nov. 1999.

RIO GRANDE DO SUL. Normas técnicas para classificação de carcaças de bovinos e bubalinos para fins de enquadramento no "Programa Carne de Qualidade", instituído pela lei $\mathrm{n}^{\circ} 10.533$, de 03 de agosto de 1995, do Governo do Estado do Rio Grande do Sul. Diário Oficial [do Estado do Rio Grande do Sul], Porto Alegre, v.54, n.188, p.42-43, 29 set. 1995.

VAZ, F.N.; RESTLE, J.; ANDREATTA, E. Peso e rendimento da carcaça de machos inteiros, terminados com diferentes níveis de concentrado na dieta. In: REUNIÃO ANUAL DA SOCIEDADE BRASILEIRA DE ZOOTECNIA, 34., 1997, Juiz de Fora. Anais. Juiz de Fora: SBZ, 1997. p.201-202. 